\title{
Radiation Therapy and Breast Cancer Risk
}

\author{
Andrea K. Ng, MD, MPH, ${ }^{a}$ and Lois B. Travis, MD, ScD, ${ }^{b}$ Boston, Massachusetts, and Rochester, New York
}

Key Words

Breast cancer, second malignant neoplasm, radiation therapy

\begin{abstract}
Exposure to ionizing radiation has clearly been established as one of the risk factors for the development of breast cancer. Much data on the relationship between radiation exposures and subsequent breast cancer are derived from atomic bomb survivors and women who received medical exposures either for diagnostic or therapeutic purposes. Although these populations differ in background breast cancer risks and the dose, quality, and timing of radiation, consistent findings include an increased risk with younger age at exposure, long latency to breast cancer development, and increasing risk with increasing radiation dose. Although therapeutic radiation is rarely used to treat benign conditions, it remains an important and effective treatment modality for a wide range of cancers. Increased knowledge of radiation-related breast cancer and modifying influences plays an important role in guiding the initial treatment approach for young women and optimizing longterm follow-up care. (JNCCN 2009;7:1121-1128)
\end{abstract}

Female breast cancer is a well-documented late effect of exposure to doses of ionizing radiation as low as 0.1 to 0.5 Sievert $(\mathrm{Sv}) .{ }^{1}$ Sievert is the unit dose equivalent and the product of the absorbed dose in gray and quality factors; in this article, Sv will be used interchangeably with Gray (Gy), the unit of absorbed dose, kerma, and specific energy imparted.

Ample data are available on radiation-associated breast cancer based on epidemiologic studies of atomic bomb survivors, ${ }^{1-9}$ and cohorts of women who under-

From the aDana-Farber Cancer Institute and Brigham and Women's Hospital, Harvard Medical School, Boston, Massachusetts, and ${ }^{b}$ Rubin Center for Cancer Survivorship and Department of Radiation Oncology, James P. Wilmot Cancer Center, University of Rochester Medical Center, Rochester, New York.

Submitted June 17, 2009; accepted for publication August 10, 2009.

The authors have disclosed that they have no financial interests, arrangements, or affiliations with the manufacturers of any products discussed in the article or their competitors.

Correspondence: Andrea K. Ng, MD, MPH, Department of

Radiation Oncology, Harvard Medical School, 75 Francis Street,

ASB1-L2, Boston, MA 02115. E-mail: ang@|roc.harvard.edu went repeated diagnostic exposure, ${ }^{10-13}$ or therapeutic radiation to treat either benign conditions ${ }^{14-24}$ or cancer. $^{25-40}$ Although the radiation administered to these populations varied in terms of quality, dose rate, fractionation, and cumulative amount, and the patients differed in terms of age at exposure and background risk, consistent findings between studies have emerged. These observations include a diminishing risk for breast cancer with increasing age at radiation exposure, and a significant relationship between increasing radiation dose and increasing breast cancer risk, although the precise shape of the dose-response curve can vary.

Preston et al. ${ }^{41}$ reviewed 8 cohorts of irradiated women, including atomic bomb survivors and 7 other groups who received medical exposures. Results from this pooled analysis suggested that radiation exposure at any age was associated with an increased risk for breast cancer. Although breast cancer excesses decreased with increasing age at exposure, significantly elevated risks persisted throughout life, with the largest excess rates occurring late in life, as background rates increase. Although a cell-killing effect for high dose-rate exposures totaling several Gy or more was suggested, the pooled analysis showed no evidence contrary to a linear dose response in the low-dose region. In subsequent studies that focused on Hodgkin lymphoma (HL) survivors who underwent radiation in therapeutic dose ranges, there was also no evidence of a downturn in risk, even in the highest dose ranges. ${ }^{34,35}$

A thorough understanding of the relation between radiation exposure and subsequent breast cancer excesses can facilitate the development of radiobiologic risk models for estimating risks in various settings. Furthermore, this information can then provide the foundation for patient counseling, and also guide recommendations for optimal breast cancer screening and prevention strategies in exposed women. 


\section{Risk in Atomic Bomb Survivors}

Epidemiologic studies on atomic bomb survivors represent a major source of data on cancer risk after small doses of radiation. The Radiation Effects Research Foundation's (RERF) extended Life Span Study (LSS) on the health effects of exposure to atomic bomb radiation published a series of reports on female breast cancer, ${ }^{2-5,7,42,43}$ with an estimated overall excess relative risk per $\mathrm{Sv}\left(\mathrm{ERR}_{1 \mathrm{~Sv}}\right)$ of $1.56 .^{3}$

An analysis of breast cancer mortality reported that women who received average doses of 0.09 , 0.75 , and $1.99 \mathrm{~Sv}$ experienced 1.05-, 2.14-, and 2.47-fold increased risks for mortality, respectively, compared with women who received zero mean doses. ${ }^{10}$ The estimated ERR ${ }_{1 \mathrm{~Sv}}$ declined with increasing age at exposure. Among survivors exposed before 20 years of age, the ERR ${ }_{1 S v}$ ranged from 2.65 to 3.94, whereas among those exposed after this age, the $E R_{1 S v}$ ranged from 0.54 to $1.33 .^{2}$ The higher dose-specific ERR among women exposed before 20 years of age could reflect the lower susceptibility to radiation carcinogenesis of terminally differentiated breast cells. In addition, older women may be more likely to have experienced a full-term pregnancy before exposure, thus reducing their breast cancer risk. A case-control study found that younger age at full-term pregnancy, multiple births, and a prolonged cumulative period of breastfeeding were protective against subsequent breast cancer among the atomic bomb survivors, whereas age at menarche and menopause did not significantly influence risk. ${ }^{5}$

A pathologic review of breast cancer in atomic bomb survivors showed that the distribution of histologic types was similar to that of de novo breast cancer in Japanese women, and did not seem dependent on dose. ${ }^{8}$ In a recent immunohistopathologic study, however, Miura et al. ${ }^{44}$ found a higher histologic grade, including larger nuclear size and higher mitotic count for breast cancers in survivors exposed to atomic bomb radiation at a closer distance. In addition, a higher incidence of both HER2 and c-myc amplification in invasive ductal carcinomas was reported among these women.

\section{Risk After Diagnostic Radiation Exposures}

The risk for breast cancer among women who underwent multiple fluoroscopic examinations during artificially induced pneumothorax to treat tuberculosis has been described in multiple studies. Patients were typically exposed to hundreds of these examinations regularly over 3 to 5 years, with an estimated dose to the breast of 0.01 to 0.1 Gy per fluoroscopy. ${ }^{12}$

In a study comparing breast cancer incidence in the Massachusetts tuberculosis fluoroscopy cohort and among atomic bomb survivors, ${ }^{12}$ the $\mathrm{ERR}_{1 \mathrm{~S}_{\mathrm{v}}}$ of breast cancer was significantly lower in the fluoroscopy cohort $(0.58$ vs. $1.55, P=.04)$. This finding was partly attributed to the low background risk for breast cancer among Japanese women. The excess absolute risk for breast cancer, however, was not significantly different between the cohorts ( 5.48 vs. 4.95 per 10,000 person-years per Sv; $P=.32$ ). Howe and McLaughlin ${ }^{10}$ described a significant effect of radiation dose on the risk for breast cancer mortality in the Canadian fluoroscopy cohort study. Women exposed to the highest mean dose category of 13.27 Sv had a 27.9-fold increased risk for breast cancer mortality compared with those who received zero mean doses.

Patients with scoliosis were historically exposed to frequent diagnostic radiographs. In the U.S. Scoliosis Cohort Study, ${ }^{45}$ the mean estimated cumulative radiation dose to the breast in exposed women was $0.12 \mathrm{~Gy}$ and mean age at exposure was 10.6 years. At an average follow-up of 40 years, the relative risk for breast cancer mortality was 1.7 (95\% CI, 1.3-2.1). The risk increased with increasing cumulative radiation dose. Patients who received breast doses of greater than $0.20 \mathrm{~Gy}$ had a standardized mortality ratio (SMR) of 3.36 ( $P$-trend, .001). Among women who received at least one radiographic examination, the estimated $\mathrm{ERR}_{1 \mathrm{~Sv}}$ was 2.7. Age at exposure was also significantly associated with breast cancer mortality. The risk was largest among patients first exposed at 10 or 11 years of age, with an SMR of 3.4 compared with 1.4 to 1.9 for younger and older ages. The dose-response relation, however, did not differ by age at first radiographic examination.

A recent study suggested that family history of breast cancer could enhance the carcinogenic effect of radiation. ${ }^{13}$ In this investigation, the overall $E R_{1 S_{v}}$ was 2.86, but was significantly higher (8.37; $P=.03$ ) among women who reported a family history of breast cancer in first- or second-degree relatives.

Mammographic screening has been shown to reduce breast cancer mortality, although its role in women aged 40 to 49 years is less clear. ${ }^{46,47}$ Current- 
ly, the U.S. Preventive Services Task Force recommends screening mammography, with or without clinical breast examination, every 1 to 2 years for women aged 40 years and older. The mean radiation dose of screening mammogram to breast has been estimated to be $2.25 \mathrm{mGy}$ per view. ${ }^{48}$ No study has yet addressed whether any association exists between this type of very low-level radiation exposure and subsequent excess breast cancers. Current estimates are largely extrapolated from populations exposed to higher doses of radiation.

In a study comparing the number of detected breast cancers with those possibly induced by mammographic screening, ${ }^{49}$ the detected/induced ratio increased with increasing age of screening. The ratios ranged from 0.47 to 2.6 among women aged 30 to 34 years, depending on radiation dose, whereas for those aged 60 to 64 years, the ratios ranged from 3.7 to 20 . Women with a family history of breast cancer, as expected, were estimated to have higher, more favorable detected/induced ratios. Based on the findings, the authors concluded that some measure of caution may be required in annual mammographic screening of women younger than 35 years.

The risk for radiation-induced breast cancer from screening mammography may be especially relevant in carriers of BRCA-1 and -2 mutations because of the young age at which examinations are initiated, combined with concerns regarding the higher risk for radiation-induced malignancy in these patients. ${ }^{50,51}$

In a recent study by Berrington de Gonzalez et al., ${ }^{52}$ the lifetime risk for radiation-induced breast cancer caused by 5 annual mammographic screenings in BRCA mutation carriers aged 40 years or younger was estimated to be 26, 20, and 13 per 10,000 women for screening examinations at ages 25 to 29,30 to 34 , and 35 to 39 years, respectively. The breast cancer mortality reduction needed to outweigh the risk associated with screening in these 3 age groups was greater than $45 \%$, greater than $12 \%$, and greater than $4 \%$, respectively. Assuming a mortality reduction from mammography of $15 \%$ to $25 \%$, the results suggested that women would derive no net benefit from annual mammographic screening at 25 to 29 years of age, a small benefit at 30 to 34 years, and some net benefit at older than 35 years.

\section{Risk After Therapeutic Radiation for Benign Conditions}

Low-dose ionizing radiation has been used to treat benign conditions, including skin hemangioma and thymic enlargement during infancy or childhood, acute postpartum mastitis, and benign breast disease. Hildreth et al. ${ }^{14}$ compared the incidence of breast cancer in a cohort of 1201 women administered radiation during infancy to treat an enlarged thymus gland with 2469 nonirradiated sisters. ${ }^{14}$ The estimated mean absorbed dose of radiation to the breast was 0.69 Gy. After a mean follow-up of 36 years, exposed women had a significantly increased 3.6-fold risk for developing breast cancer compared with siblings. A significant linear dose-response relationship was observed.

Similarly, increased risk for developing breast cancer was shown in women irradiated for skin hemangiomas during infancy. In a cohort study in Sweden including 17,202 women, the median absorbed breast dose was $0.05 \mathrm{~Gy}$ (average dose, 0.29 Gy; range, 0-35.8 Gy). ${ }^{17}$ Compared with the normal matched population, the relative risk for developing breast cancer was 1.2 (95\% CI, 1.09-1.36) and the $E R_{1 S v}$ was estimated at 0.35 , which is lower than that observed in most other studies on radiationrelated cancer. In the pooled analysis by Preston et al., ${ }^{41}$ a lower excess risk in the hemangioma cohorts was similarly observed, suggesting a possible ameliorating dose-rate effect for protracted low doserate exposure in women irradiated for skin hemangiomas, which was typically administered with radium-226 applicators.

One study showed that 601 women who underwent radiation therapy to treat acute postpartum mastitis had a significantly increased 3.2-fold risk for developing breast cancer at a mean follow-up of 29 years compared with control subjects who did not receive radiation therapy. ${ }^{20}$ The risk increased with increasing radiation dose in a linear fashion. Unlike other studies on radiation-related breast cancer, however, age at exposure did not significantly influence breast cancer risk. This observation was hypothesized to reflect the increased susceptibility to radiation of proliferating breast tissues stimulated by hormones during pregnancy and lactation regardless of age at treatment. Similarly, an increased risk for breast cancer was observed in women who were irradiated for benign breast disease. 
In a cohort study of 1216 women with benign breast disease treated with radiation therapy, ${ }^{23}$ the relative risk for breast cancer was significantly increased at 3.26 compared with the normal matched population. The relative risk was higher in women younger than 40 years at exposure than in those 40 years or older (relative risk, 3.92 vs. 2.54). A radiation dose-response relationship was also observed, although it was significant only among women younger than 25 years of age at exposure. ${ }^{24}$

\section{Risk After Therapeutic Radiation to Treat Cancer}

Many data on breast cancer risk after radiotherapy derive from women given chest irradiation to treat HL during childhood or young adulthood. ${ }^{34-40}$ Increasing literature also exists on the risk for contralateral breast cancer after either breast-conserving therapy with lumpectomy and radiation therapy or postmastectomy chest wall irradiation. ${ }^{25-27,29-33}$ As in breast cancer after exposure to low doses of radiation, breast cancer after high-dose therapeutic radiation therapy is characterized by a higher risk with younger age at exposure, a long latency period, and a significant radiation dose-response relationship.

\section{Breast Cancer After Radiation Therapy for HL and Childhood Cancer}

Breast cancer is one of the most common second primary tumors in childhood cancer survivors whose treatment included chest radiation therapy. In a study of survivors of childhood HL conducted by the Late Effects Study Group, ${ }^{40}$ the relative risk of breast cancer was 56.7 (95\% CI, 40.5-77.3) with a median latency of 18.1 years (range, 4.3-28.3 years). In another multi-institutional follow-up study of HL survivors, ${ }^{53}$ the relative risk for breast cancer was 37.2 (95\% CI, 25.0-53.6) and the absolute excess risk was 18.6 per 10,000 person-years. In addition, $34 \%$ of patients were diagnosed with bilateral disease. The mean time to development of breast cancer after HL was 18.7 years; among women who developed bilateral breast cancer, the average interval between the first and second cancer was 23 months.

Based on data from the Childhood Cancer Survivor Study (CCSS), Kenney et al. ${ }^{38}$ described the risk for breast cancer in 6068 female patients. Ninety-five women ( $68 \%$ of whom were survivors of pediatric HL) were diagnosed with 111 confirmed cases of breast cancer. Girls who were treated between ages 5 and 9 years did not subsequently experience a significantly increased risk for breast cancer, whereas significant excesses were observed among girls treated at 10 years of age or older. The authors suggested that, in contrast to prepubertal breast tissues, proliferating and developing breast tissues may be more sensitive to the tumorigenic effects of radiation.

In studies focused largely on survivors of adult HL, second primary breast cancer also emerged as one of the most common solid tumors. Studies have consistently shown that younger age at radiation treatment is associated with a significantly larger risk for breast cancer. ${ }^{36,54-56}$ In a population-based cohort study, Hodgson et al..$^{55}$ reported that the absolute risks for breast cancer in women diagnosed with HL at ages 15 to 25 years were 34 to 47 per 10,000 person years at 10 years, which was higher than the absolute risks among women in the general population between ages 50 and 54 years, a standard age when mammographic screening is recommended. Given this considerable risk for breast cancer, informed counseling for long-term survivors of HL is critical.

Travis et al. ${ }^{37}$ developed estimates of the cumulative absolute risk for breast cancer, taking into account age and calendar year of HL diagnosis, age at counseling, baseline breast cancer incidence rates, radiation dose and chemotherapy, and competing causes of mortality. For example, for an HL survivor who received a chest radiation dose of 40 Gy or greater at 25 years of age without undergoing alkylating agent therapy, the cumulative absolute risk for breast cancer was estimated to be $1.4 \%$ after 10 years, $11 \%$ after 20 years, and $29 \%$ after 30 years.

Several case-control studies have clearly documented a convincing radiation dose-response relation for breast cancer after HL. In a large international case-control study of HL survivors, including 105 cases of breast cancer and 266 matched controls, Travis et al..$^{35}$ estimated radiation dose to the area of the breast where the tumor developed (and a comparable area in matched controls) for each case-control set. Breast cancer risk increased significantly with increasing radiation dose, reaching 8-fold for the highest category (median dose, 42 Gy) compared with the lowest dose group ( $<4$ Gy; $P$ trend for dose, $<.001)$. A separate Dutch study ${ }^{34}$ similarly showed a significant radiation dose-response relationship, with most patients also included in the international 
investigation. The CCSS recently published a casecontrol study of 120 cases of breast cancer $(65 \%$ in survivors of HL) matched with 464 controls according to age and time since initial cancer diagnosis. ${ }^{57}$ Again, a significant linear radiation dose-response was observed ( $P$ trend, $<.0001$ ), with an estimated relative risk for breast cancer of 6.4 at $20 \mathrm{~Gy}$ and 11.8 at $40 \mathrm{~Gy}$.

Early menopause seems to have a protective effect on radiation-associated breast cancer, because of either alkylating agent chemotherapy or radiation dose greater than 5 Gy administered to the ovaries. ${ }^{34,35}$ The Dutch study specifically showed that the breast cancer risk reduction associated with chemotherapy was secondary to the high number of women who developed premature menopause. ${ }^{34}$ This casecontrol study showed that women who experienced menopause at 19 to 35 years of age had a significantly decreased risk for developing breast cancer compared with women who did not undergo premature menopause (relative risk, 0.06; 95\% CI, 0.01-0.45).34 Results suggest that ovarian hormones play an important role in promoting tumorigenesis once an initiating event is produced by radiation. However, alkylating chemotherapy's protective effect against breast cancer is less evident in survivors of childhood $\mathrm{HL},{ }^{38,57}$ which may be related to the larger reserve of oocytes and follicles in girls, and perhaps differences in types of alkylating agents and doses between pediatric and adult patients.

The data on breast cancer risk after radiotherapy for HL were based on patients treated in an era during which large treatment fields and very high radiation doses were used. In contrast, radiation treatment fields are significantly smaller, with the current standard of involved-field radiation therapy given as part of combined modality therapy. Moreover, current studies are also exploring the effect of further reductions in radiation treatment dose for $\mathrm{HL}$, and a trend exists toward the application of involved-node radiation therapy, both of which will result in additional reductions in the exposure of normal tissue to radiation. ${ }^{58}$ Therefore, patients with HL who undergo radiotherapy in the modern treatment era will probably incur a lower risk for breast cancer.

For survivors of HL, NCCN recommends mammogram/breast MRI screening 8 to 10 years after irradiation or by 40 years of age. The American Cancer Society currently recommends yearly breast MRI imaging as an adjunct to mammography in women who underwent chest radiation between 10 and 30 years of age. ${ }^{59}$ Prospective trials evaluating the role of breast MRI screening in women who underwent mantle or mediastinal radiotherapy for HL are ongoing at the Dana-Farber Cancer Institute and M. D. Anderson Cancer Center.

\section{Contralateral Breast Cancer After Radiation Therapy}

In an overview of randomized trials on surgery with or without radiotherapy for early-stage breast cancer, the Early Breast Cancer Trialists' Collaborative Group (EBCTCG) showed that improvement in locoregional control with the addition of radiation therapy resulted in a significant reduction in 15. year breast cancer-specific and overall mortality. ${ }^{60}$ Therefore, radiation therapy is expected to continue to play an important role in the curative treatment for breast cancer, either as part of breast conserving therapy or given as postmastectomy chest wall irradiation in high-risk patients.

One long-term effect of radiation therapy is an increased risk for second malignancy, including risk for contralateral breast cancer, which accounts for $40 \%$ of all second tumors among women with breast cancer, with a 25 -year cumulative risk of $6.9 \%{ }^{61} \mathrm{Al}$ though these excesses are largely related to preexisting breast cancer risk factors, prior radiation therapy, especially treatment at a young age, may contribute to the increased risk. ${ }^{28-30,62}$

In early case-control studies by Storm et al..$^{33}$ and Boice et al., ${ }^{62}$ the overall relative risk for contralateral breast cancer was not significantly increased after radiation therapy (relative risk, 1.2; 95\% CI, 0.94, 1.2). However, among women younger than 45 years at irradiation, Boice et al. ${ }^{62}$ showed that the relative risk for breast cancer was significantly elevated at 1.6 (95\% CI, 1.1-2.4).

A recent Dutch study ${ }^{32}$ assessed the long-term risk for contralateral breast cancer in 7425 survivors of breast cancer. Similar to the earlier studies, the overall relative risk for contralateral breast cancer for those who underwent radiation therapy was not significantly elevated compared with those who did not (after adjusting for family and smoking histories). However, in patients who underwent radiation therapy before 35 years of age, the hazard ratio for contralateral breast cancer was 1.78 (95\% CI, 0.853.72), and decreased to 1.09 (95\% CI, 0.82-1.45) 
among women irradiated at 45 years or age or older.

Stovall et al. ${ }^{25}$ conducted a multi-institutional case-control study of 708 women with contralateral breast cancer, matched with 1399 controls. Among women younger than 40 years, those who received more than 1 Gy of radiation had a 2.5-fold (95\% CI, 1.4-4.5) greater risk than unexposed women, whereas no excess risk was observed for women older than 40 years treated with radiotherapy. In contrast, the EBCTCG study ${ }^{60}$ found a significantly increased risk for contralateral breast cancer after radiotherapy (relative risk, 1.4; $P=.00001$ ), and remained statistically significant among women older than 50 years at treatment (relative risk, 1.3; $P=.002$ ).

Studies have also shown that the risk for contralateral breast cancer in breast cancer survivors is significantly associated with radiation dose, although the association was mainly limited to younger women. In the case-control study by Stovall et al., ${ }^{25}$ women younger than 40 years had an excess relative risk of 0.6 per Gy (95\% CI, 0.1-1.5), and those younger than 40 years who had at least a 5 -year latency had an excess relative risk of 1 per Gy $(95 \%$ CI, 0.1-3.0). In the Dutch study, ${ }^{32}$ among women younger than 45 years at irradiation, a significant dose-response relationship was also observed, with a linear excess relative risk of 0.21 per Gy increase ( $P$ trend, .03). The relationship was stronger for risk of medially located contralateral breast cancer, with a linear excess relative risk of 0.37 per $G y$ ( $P$-trend, $.01)$. In addition, women treated with postlumpectomy radiation (which was associated with higher doses to the contralateral breast) had a significantly 1.5-fold increased risk for contralateral breast cancer compared with patients treated with postmastectomy radiation therapy.

\section{Conclusions}

Epidemiologic data on historical cohorts of women exposed to various forms of ionizing radiation have provided a valuable foundation on the relationship between radiation exposure and subsequent breast cancer risk. Although radiation therapy is now rarely used to treat benign conditions, it remains a key modality in the management of many patients with cancer, including children and young adults. The cumulative risk for subsequent breast cancer in these patients ${ }^{37}$ should be taken into consideration both during initial treatment decisions and implementation of follow-up strategies. Future directions include the development of radiation techniques and treatment strategies to minimize dose to breast tissue, and the identification of underlying susceptibility factors $^{50-52}$ and modifying influences..$^{5,13,16,34,35,63}$ Moreover, the molecular and pathologic features ${ }^{44,64}$ of radiation-associated breast cancer should continue to be characterized. Furthermore, in this era of rapidly advancing molecular technologies, which include genome-wide association studies and next-generation sequencing, application of these techniques might help identify women at highest risk for radiation-associated cancer. In the interim, breast cancer screening and prevention programs should be implemented for long-term cancer survivors who undergo chest radiotherapy, along with efforts directed toward educating patients and health care providers. ${ }^{65}$

\section{References}

1. Land CE. Studies of cancer and radiation dose among atomic bomb survivors. The example of breast cancer. JAMA 1995;274:402-407.

2. Land CE, Tokunaga $M$, Koyama $K$, et al. Incidence of female breast cancer among atomic bomb survivors, Hiroshima and Nagasaki, 1950-1990. Radiat Res 2003;160:707-717.

3. Tokunaga M, Land CE, Tokuoka S, et al. Incidence of female breast cancer among atomic bomb survivors, 1950-1985. Radiat Res 1994;138:209-223.

4. Land CE, Hayakawa N, Machado SG, et al. A case-control interview study of breast cancer among Japanese A-bomb survivors. II. Interactions with radiation dose. Cancer Causes Control 1994;5:167-176.

5. Land CE, Hayakawa N, Machado SG, et al. A case-control interview study of breast cancer among Japanese A-bomb survivors. I. Main effects. Cancer Causes Control 1994;5:157-165.

6. Land CE, Tokunaga M, Tokuoka S, Nakamura N. Early-onset breast cancer in A-bomb survivors. Lancet 1993;342:237.

7. Tokunaga M, Land CE, Tokuoka S. Follow-up studies of breast cancer incidence among atomic bomb survivors. J Radiat Res (Tokyo) 1991;32(Suppl):201-211.

8. Tokuoka S, Asano M, Yamamoto T, et al. Histologic review of breast cancer cases in survivors of atomic bombs in Hiroshima and Nagasaki, Japan. Cancer 1984;54:849-854.

9. Tokunaga $M$, Land CE, Yamamoto $T$, et al. Breast cancer in Japanese A-bomb survivors. Lancet 1982;2:924.

10. Howe GR, McLaughlin J. Breast cancer mortality between 1950 and 1987 after exposure to fractionated moderate-dose-rate ionizing radiation in the Canadian fluoroscopy cohort study and a comparison with breast cancer mortality in the atomic bomb survivors study. Radiat Res 1996;145:694-707.

11. Boice JD Jr, Preston D, Davis FG, Monson RR. Frequent chest $\mathrm{X}$-ray fluoroscopy and breast cancer incidence among tuberculosis patients in Massachusetts. Radiat Res 1991;125:214-222. 
Radiation Therapy and Breast Cancer Risk

12. Little MP, Boice JD Jr. Comparison of breast cancer incidence in the Massachusetts tuberculosis fluoroscopy cohort and in the Japanese atomic bomb survivors. Radiat Res 1999;151:218-224.

13. Ronckers CM, Doody MM, Lonstein JE, et al. Multiple diagnostic $\mathrm{X}$-rays for spine deformities and risk of breast cancer. Cancer Epidemiol Biomarkers Prev 2008;17:605-613.

14. Hildreth NG, Shore RE, Dvoretsky PM. The risk of breast cancer after irradiation of the thymus in infancy. $\mathrm{N}$ Engl J Med 1989;321:1281-1284.

15. Hildreth NG, Shore RE, Hempelmann LH. Risk of breast cancer among women receiving radiation treatment in infancy for thymic enlargement. Lancet 1983;2:273.

16. Holmberg E, Holm LE, Lundell M, et al. Excess breast cancer risk and the role of parity, age at first childbirth and exposure to radiation in infancy. Br J Cancer 2001;85:362-366.

17. Lundell M, Mattsson $A$, Karlsson P, et al. Breast cancer risk after radiotherapy in infancy: a pooled analysis of two Swedish cohorts of 17,202 infants. Radiat Res 1999;151:626-632.

18. Lundell M, Mattsson A, Hakulinen T, Holm LE. Breast cancer after radiotherapy for skin hemangioma in infancy. Radiat Res 1996;145:225-230.

19. Lindberg S, Karlsson P, Arvidsson B, et al. Cancer incidence after radiotherapy for skin haemangioma during infancy. Acta Oncol 1995;34:735-740.

20. Shore RE, Hildreth N, Woodard E, et al. Breast cancer among women given X-ray therapy for acute postpartum mastitis. J Natl Cancer Inst 1986;77:689-696.

21. Logan WW, Mansur PS, Cullinan A, et al. Increased incidence of breast carcinoma in patients with irradiation for post-partum mastitis: a screening situation. J Surg Oncol 1979;11:239-242.

22. Prince MM, Hildreth NG. The influence of potential biases on the risk of breast tumors among women who received radiotherapy for acute postpartum mastitis. J Chronic Dis 1986;39:553-560.

23. Mattsson A, Ruden BI, Palmgren J, Rutqvist LE. Dose- and timeresponse for breast cancer risk after radiation therapy for benign breast disease. Br J Cancer 1995;72:1054-1061.

24. Mattsson A, Ruden BI, Hall P, et al. Radiation-induced breast cancer: long-term follow-up of radiation therapy for benign breast disease. J Natl Cancer Inst 1993;85:1679-1685.

25. Stovall M, Smith SA, Langholz BM, et al. Dose to the contralateral breast from radiotherapy and risk of second primary breast cancer in the WECARE study. Int J Radiat Oncol Biol Phys 2008;72:10211030.

26. Innos $\mathrm{K}$, Horn-Ross PL. Risk of second primary breast cancers among women with ductal carcinoma in situ of the breast. Breast Cancer Res Treat 2008;111:531-540.

27. Yadav BS, Sharma SC, Patel FD, et al. Second primary in the contralateral breast after treatment of breast cancer. Radiother Oncol 2008;86:171-176.

28. Kirova YM, Gambotti L, De Rycke Y, et al. Risk of second malignancies after adjuvant radiotherapy for breast cancer: a large-scale, single-institution review. Int J Radiat Oncol Biol Phys 2007;68:359-363.

29. Hill-Kayser CE, Harris EE, Hwang WT, Solin LJ. Twenty-year incidence and patterns of contralateral breast cancer after breast conservation treatment with radiation. Int J Radiat Oncol Biol Phys 2006;66:1313-1319.

30. Gao X, Fisher SG, Emami B. Risk of second primary cancer in the contralateral breast in women treated for early-stage breast cancer: a population-based study. Int J Radiat Oncol Biol Phys 2003;56:1038-1045.

31. Fowble B, Hanlon A, Freedman G, et al. Second cancers after conservative surgery and radiation for stages I-II breast cancer: identifying a subset of women at increased risk. Int J Radiat Oncol Biol Phys 2001;51:679-690.

32. Hooning MJ, Aleman BM, Hauptmann M, et al. Roles of radiotherapy and chemotherapy in the development of contralateral breast cancer. J Clin Oncol 2008;26:5561-5568.

33. Storm HH, Andersson M, Boice JD Jr, et al. Adjuvant radiotherapy and risk of contralateral breast cancer. J Natl Cancer Inst 1992;84:1245-1250.

34. van Leeuwen FE, Klokman WJ, Stovall M, et al. Roles of radiation dose, chemotherapy, and hormonal factors in breast cancer following Hodgkin's disease. J Natl Cancer Inst 2003;95:971-980.

35. Travis LB, Hill DA, Dores GM, et al. Breast cancer following radiotherapy and chemotherapy among young women with Hodgkin disease. JAMA 2003;290:465-475.

36. Aisenberg AC, Finkelstein DM, Doppke KP, et al. High risk of breast carcinoma after irradiation of young women with Hodgkin's disease. Cancer 1997;79:1203-1210.

37. Travis LB, Hill D, Dores GM, et al. Cumulative absolute breast cancer risk for young women treated for Hodgkin lymphoma. J Natl Cancer Inst 2005;97:1428-1437.

38. Kenney LB, Yasui Y, Inskip PD, et al. Breast cancer after childhood cancer: a report from the Childhood Cancer Survivor Study. Ann Intern Med 2004;141:590-597.

39. Constine LS, Tarbell N, Hudson MM, et al. Subsequent malignancies in children treated for Hodgkin's disease: associations with gender and radiation dose. Int J Radiat Oncol Biol Phys 2008;72:24-33.

40. Bhatia S, Yasui Y, Robison LL, et al. High risk of subsequent neoplasms continues with extended follow-up of childhood Hodgkin's disease: report from the Late Effects Study Group. J Clin Oncol 2003;21:4386-4394.

41. Preston DL, Mattsson A, Holmberg E, et al. Radiation effects on breast cancer risk: a pooled analysis of eight cohorts. Radiat Res 2002;158:220-235.

42. Tokunaga $M$, Land $C E$, Yamamoto $T$, et al. Incidence of female breast cancer among atomic bomb survivors, Hiroshima and Nagasaki, 1950-1980. Radiat Res 1987;112:243-272.

43. Tokunaga M, Norman JE Jr, Asano M, et al. Malignant breast tumors among atomic bomb survivors, Hiroshima and Nagasaki, 1950-74. J Natl Cancer Inst 1979;62:1347-1359.

44. Miura S, Nakashima M, Ito M, et al. Significance of HER2 and C-MYC oncogene amplifications in breast cancer in atomic bomb survivors: associations with radiation exposure and histologic grade. Cancer 2008;112:2143-2151.

45. Morin Doody M, Lonstein JE, Stovall M, et al. Breast cancer mortality after diagnostic radiography: findings from the U.S. Scoliosis Cohort Study. Spine 2000;25:2052-2063.

46. Moss SM, Cuckle H, Evans A, et al. Effect of mammographic screening from age 40 years on breast cancer mortality at 10 years' follow-up: a randomised controlled trial. Lancet 2006;368:20532060.

47. Djulbegovic B, Lyman GH. Screening mammography at 40-49 years: regret or no regret? Lancet 2006;368:2035-2037.

48. Young KC, Burch A, Oduko JM. Radiation doses received in the UK Breast Screening Programme in 2001 and 2002. Br J Radiol 2005;78:207-218. 
49. Law J, Faulkner K. Cancers detected and induced, and associated risk and benefit, in a breast screening programme. Br J Radiol 2001;74:1121-1127.

50. Broeks A, Braaf LM, Huseinovic A, et al. Identification of women with an increased risk of developing radiation-induced breast cancer: a case only study. Breast Cancer Res 2007;9:R26.

51. Cardis E, Hall J, Tavtigian SV. Identification of women with an increased risk of developing radiation-induced breast cancer. Breast Cancer Res 2007;9:106.

52. Berrington de Gonzalez A, Berg CD, Visvanathan K, Robson M. Estimated risk of radiation-induced breast cancer from mammographic screening for young BRCA mutation carriers. J Natl Cancer Inst 2009;101:205-209.

53. Basu SK, Schwartz C, Fisher SG, et al. Unilateral and bilateral breast cancer in women surviving pediatric Hodgkin's disease. Int J Radiat Oncol Biol Phys 2008;72:34-40.

54. Ng AK, Bernardo MV, Weller E, et al. Second malignancy after Hodgkin disease treated with radiation therapy with or without chemotherapy: long-term risks and risk factors. Blood 2002;100:1989-1996.

55. Hodgson DC, Gilbert ES, Dores GM, et al. Long-term solid cancer risk among 5-year survivors of Hodgkin's lymphoma. J Clin Oncol 2007;25:1489-1497.

56. Hancock SL, Tucker MA, Hoppe RT. Breast cancer after treatment of Hodgkin's disease. J Natl Cancer Inst 1993;85:25-31.

57. Inskip P, Robison L, Stovall M, et al. Radiation dose and breast cancer risk in the childhood cancer survivor study. J Clin Oncol 2009;27:3901-3907.
58. Girinsky $T$, van der Maazen R, Specht L, et al. Involved-node radiotherapy (INRT) in patients with early Hodgkin lymphoma: concepts and guidelines. Radiother Oncol 2006;79:270-277.

59. Saslow D, Boetes C, Burke W, et al. American Cancer Society guidelines for breast screening with MRI as an adjunct to mammography. CA Cancer J Clin 2007;57:75-89.

60. Clarke M, Collins R, Darby S, et al. Effects of radiotherapy and of differences in the extent of surgery for early breast cancer on local recurrence and 15-year survival: an overview of the randomised trials. Lancet 2005;366:2087-2106.

61. Curtis R, Freedman D, Ron E, et al. New malignancies among cancer survivors: SEER Cancer Registries, 1973-2000. National Cancer Institute. NIH Publication No. 05-5302. Bethesda, MD, 2006.

62. Boice JD Jr, Harvey EB, Blettner M, et al. Cancer in the contralateral breast after radiotherapy for breast cancer. N Engl J Med 1992;326:781-785.

63. Hill DA, Gilbert E, Dores GM, et al. Breast cancer risk following radiotherapy for Hodgkin lymphoma: modification by other risk factors. Blood 2005;106:3358-3365.

64. Castiglioni F, Terenziani M, Carcangiu ML, et al. Radiation effects on development of HER2-positive breast carcinomas. Clin Cancer Res 2007;13:46-51.

65. Oeffinger KC, Ford JS, Moskowitz CS, et al. Breast cancer surveillance practices among women previously treated with chest radiation for a childhood cancer. JAMA 2009;301:404-414. 